\title{
The use of ex vivo ovary culture for assessment of alterations in steroidogenesis following neonatal exposure to poly(ethylene glycol)-block- polylactide methyl ether or titanium dioxide nanoparticles in Wistar rats
}

\author{
Sona Scsukova ${ }^{1}$, Alzbeta Bujnakova Mlynarcikova ${ }^{1}$, Eva Rollerova ${ }^{2}$ \\ ${ }^{1}$ Institute of Experimental Endocrinology, Biomedical Research Center, Slovak Academy of Sciences, Bratislava, Slovakia; \\ ${ }^{2}$ Department of Toxicology, Faculty of Public Health, Slovak Medical University in Bratislava, Bratislava, Slovakia \\ E-mail: sona.scsukova@hotmail.com
}

Objectives. Rapid development and widespread application of different types of nanoparticles (NPs) may result in increased exposure of humans and animals to NPs. Recently, reproductive toxicity due to NP exposure has become a major component of risk assessment. Current data have suggested that NPs may pose adverse effects on male and female reproductive health by altering normal testis and ovarian structure, and sex hormone levels. To detect possible alterations in steroidogenesis in adult and infantile rats following neonatal exposure to polymeric poly(ethylene glycol)-block-polylactide methyl ether (PEG- $b$-PLA) or titanium dioxide $\left(\mathrm{TiO}_{2}\right) \mathrm{NPs}$, whole ovary cultures were used.

Methods. Newborn female Wistar rats were intraperitoneally (i.p.) injected daily with two different doses of PEG- $b$-PLA NPs (20 and $40 \mathrm{mg} / \mathrm{kg}$ body weight, b.w.) or $\mathrm{TiO}_{2} \mathrm{NPs}$ (1\% LD50 $\mathrm{TiO}_{2}=59.2 \mu \mathrm{g} / \mathrm{kg}$ b.w. and $10 \% \mathrm{LD}_{0} \mathrm{TiO}_{2}=592 \mu \mathrm{g} / \mathrm{kg}$ b.w.) from postnatal day 4 (PND 4 ) to PND 7. The ovaries were collected on PND73 and PND15 of PEG-b-PLA- and $\mathrm{TiO}_{2}$ NP-treated rats, respectively, and their corresponding control animals. Minced ovaries were cultured in vitro in the absence (basal conditions) or presence of gonadotropins (follicle-stimulating hormone, FSH and luteinizing hormone, LH) and insulin-like growth factor-1 (IGF-1) (stimulated conditions) for 6 days. At indicated time intervals, culture media were collected for steroid hormone (progesterone, estradiol) analysis by specific radioimmunoassay (RIA) and enzyme-linked immunosorbent assay (ELISA) kits.

Results. Basal progesterone and estradiol secretion by ovaries from adult rats (PND73) were significantly decreased $(\mathrm{p}<0.01)$ in both PEG- $b$-PLA-treated groups after 3 days and 1 day of ex vivo ovary culture, respectively, compared with control group. With the presence of FSH/LH and IGF-1 in the culture medium, progesterone and estradiol production significantly increased $(\mathrm{p}<0.001)$ compared to basal levels. Stimulated progesterone production was significantly decreased $(\mathrm{p}<0.05)$ in PEG-b-PLA40-treated group after 3 days of culture compared with controls. After ex vivo culture of rat ovaries collected on PND15, basal progesterone and estradiol levels measured in the culture media did not differ between control and both $\mathrm{TiO}_{2} \mathrm{NP}$-treated groups. The ovaries from rats neonatally exposed to both doses of $\mathrm{TiO}_{2} \mathrm{NPs}$ failed to respond to FSH/IGF stimulation in progesterone secretion at all time intervals.

Conclusions. The obtained results indicate that neonatal exposure to NPs in female rats may alter ovarian steroidogenic output (steroid hormone secretion) and thereby might subsequently induce perturbation of mammalian reproductive functions. Possible mechanisms (induction of oxidative stress, inflammation) of adverse effects of NPs on ovarian function should be further elucidated.

Key words: polymeric nanoparticles, PEG-b-PLA, titanium dioxide, ovary, progesterone, estradiol, reproductive toxicity

Corresponding author: Sona Scsukova, PhD., Institute of Experimental Endocrinology, Biomedical Research Center, Slovak Academy of Sciences, Dubravska cesta 9, 84505 Bratislava, Slovakia; phone: +421-2-32295900; e-mail: sona.scsukova@hotmail.com. 
With the rapid development and widespread use of nanoparticles (NPs) in many industrial and biomedical applications, the environmental and occupational exposure of humans and animals to NPs is dramatically increasing.

Over the past few decades, polymeric micelles have attracted increasing attention as the carriers for various biologically active substances such as drugs, proteins and nucleic acids (De Jong and Borm 2008; Moritz and Geszke-Moritz 2015; Wang et al. 2017; Casalini et al. 2019). Polymeric micelles exhibit good biodegradability and biocompatibility; they offer numerous attractive features such as targeted drug delivery, sustained release or prolonged circulation half-life (Semete et al 2010; Moritz and Geszke-Moritz 2015). An amphiphilic block copolymer (ABC) poly(ethylene glycol)-block-poly(lactic acid) (PEG- $b$ PLA), a U.S. Food and Drug Administration (FDA)approved material, is composed of a hydrophilic PEG block and a hydrophobic PLA block, which can selfassemble into micelles in water. It is one of the most commonly used biodegradable polymers for drug encapsulation, drug solubilization and drug delivery (Xiao et al. 2010; Shin et al. 2012; Shen et al. 2015; Cho et al. 2016). PEGylated liposomal doxorubicin (PLD) has been already approved for treatment of advanced ovarian cancer patients failing first-line platinumbased treatment (Ferrandina et al. 2010).

Metal and metal-oxide NPs are two of the most prevalent types of manufactured NPs used in medicine, consumer products and industrial settings. Nano-sized titanium dioxide $\left(\mathrm{TiO}_{2} \mathrm{NP}\right)$ is manufactured worldwide in large quantities and is extensively applied in biological tissue engineering materials, food additives, cosmetics, and sunscreens. Therefore, human exposure may occur through ingestion and dermal penetration, or through inhalation route, during both the manufacturing process and use (Weir et al. 2012; Rompelberg et al. 2016). In spite of the extensively use of $\mathrm{TiO}_{2} \mathrm{NPs}$, the biological effects and the cellular response mechanisms of $\mathrm{TiO}_{2} \mathrm{NPs}$ are still not completely elucidated and thus a deep understanding of the toxicological profile of this compound is required (Grande and Tucci 2016).

The results of recent studies have reported that NPs may pose adverse effects on male and female reproductive health by altering normal testis and ovarian structure, spermatogenesis and sperm quality, oogenesis, follicle maturation and sex hormone levels (Taylor et al. 2012; Iavicoli et al. 2013; Larson et al. 2014; Hou and Zhu 2017). The adverse effects of polymeric NPs have not been extensively studied and only few studies have focused on their effects on reproductive system to date (Rollerova et al. 2015a; Scsukova et al. 2015, 2017). Numerous studies to date have demonstrated that $\mathrm{TiO}_{2} \mathrm{NPs}$ penetrates through the digestive system and possibly the blood circulation, leading to accumulation in the ovary and consequent reproductive toxicity (Shi et al. 2013; Das et al. 2016; Alaee et al. 2017; Hong et al. 2017). However, the mechanisms underlying the toxic effects of $\mathrm{TiO}_{2}$ NPs on the female reproductive system remain to be established.

A great attention is dedicated to an establishment of alternative approaches (in vitro 3D and organ culture) for reproductive toxicity evaluation, because they can reduce the number of experimental animals used for in vivo studies. In vitro ovary and follicle culture models mimic the in vivo environment, allow the possibility of varying culture parameters in a highly controlled manner. Potential endpoints include studying the mechanisms of action of different toxicants and their contribution to follicular cell damage (Stefansdottir el al. 2014).

In the present study, whole ovary culture was used as an in vitro assay for analyzing ovarian steroidogenic activity following in vivo neonatal PEG- $b$-PLA and $\mathrm{TiO}_{2} \mathrm{NPs}$ exposure in adult and infantile female Wistar rats.

\section{Materials and methods}

\section{Experiment 1: PEG-b-PLA NP exposure.}

Preparation and characterization of PEG- $b$-PLA NPs. As the present experiment is an integral part of a toxicological study (Rollerova et al. 2015a), minimal physicochemical characterization of PEG- $b$-PLA was carried out according to recommended requirements for toxicological studies and safety/hazard/risk assessment (Wang et al. 2013).

Fresh NP micelles of PEG- $b$-PLA were prepared by modified solvent evaporation method according to $\mathrm{Du}$ et al. (2009) and Shin et al. (2009). Briefly, copolymer PEG- $b$-PLA [CH3O (CH2CH2O)x(COCHCH3O)yH, PEG average $\mathrm{Mn}=350 \mathrm{~g} / \mathrm{mol}$, PLA average $\mathrm{Mn}=$ $1000 \mathrm{~g} / \mathrm{mol}$, CAS 9004-74-4; Sigma-Aldrich, Germany] (20 mg) was dissolved in $2 \mathrm{ml}$ of tetrahydrofuran (THF; anhydrous, inhibitor free, purity $\geq 99.9 \%$; Sigma-Aldrich, Germany) and stirred for $2 \mathrm{~h}$ at room temperature (RT). Under moderate stirring (100 rpm, MR Hei-Standard Heidolph, Germany), the ultrapurified water $(10 \mathrm{ml})$ (MiliporeMili-Q Synthesis, $18.5 \mathrm{M} \Omega$ ) was added dropwise. Two hours later, THF was evaporated under mild vacuum (rotating evaporator LABOROTA 4010- 
digital, Heidolph, Germany) for $1 \mathrm{~h}$ at $48^{\circ} \mathrm{C}$ to obtain polymer micelles. After THF evaporation, water was added to the suspension to obtain the final PEG- $b$ PLA concentration of $2 \mathrm{mg} / \mathrm{ml}$. Immediately before administration, PEG- $b$-PLA suspension was vortexed at the highest speed for $1 \mathrm{~min}$.

Physical particle size, general state of agglomeration/aggregation and morphology of PEG- $b$-PLA suspension were determined by transmission electron micrography (TEM) using JEM 1200 microscope (JOEL, Tokyo, Japan) with $120 \mathrm{kV}$ voltage. Zeta potential was measured by Nicomp Submicron Particle Sizer Autodilute Model 380 (Santa Barbara, CA, USA) using the electrophoretic light scattering (ELS) method. Size distribution of PEG- $b$-PLA was evaluated by dynamic light scattering (DLS) with a NICOMP TM 380 ZLS Particle Sizer (Santa Barbara, CA, USA).

TEM demonstrated spherical shape of NPs and the average primary particle size suspended in deionized water about $50 \mathrm{~nm}$. Zeta potential value was $28.73 \pm 1.44 \mathrm{mV}$. Micelle dispersion resulted in size distribution with two main peaks of secondary particle sizes, averaged as $64.9 \pm 10.5 \mathrm{~nm}$ and 911.4 $\pm 177.6 \mathrm{~nm}$, which indicates that the PEG- $b$ PLA micelles were aggregated in solution. Similar to the findings in Shin et al. (2009), the PEG- $b$-PLA micelles were stable for $24 \mathrm{~h}$ at ambient temperature as monitored by TEM. More details are presented in the paper by Rollerova et al. (2015a).

Study design. The study was carried out on one generation of the neonatal rat model described by the Newbold's group representing an ontogenic model consistent with the developmental stage from 0 to 28 postnatal days in human infants (Newbold et al. 2007; Picut et al. 2015). In the present study, 34 female neonates were used. Parent specific pathogen free Wistar rats were obtained from Breeding Facility Masaryk University Brno (Czech Republic). To simulate intravenous administration of PEG- $b$-PLA micelles to humans, intraperitoneal (i.p.) administration was used. Daily, on postnatal day 4 (PND4) to PND7, neonatal rats were i.p. injected with one of two doses of PEG- $b$-PLA NPs [20 mg/kg body weight (b.w.), $\mathrm{n}=13$ or $40 \mathrm{mg} / \mathrm{kg}$ of b.w., $\mathrm{n}=10$ ]. In negative control group $(n=12)$, animals were injected with ultrapurified water after evaporation of THF (vehicle used in NP preparation). On PND73 (adult rats), the ovaries were collected for ex vivo culture.

Ex vivo ovary culture. The ovaries collected on PND73 were minced with scissors into 3-4 pieces and cultured in vitro in DMEM/F12 (Dulbecco's Modified Eagle's Medium/Nutrient Mixture F-12 Ham with $1 \mathrm{mM}$ L-glutamine and HEPES; Sigma-Aldrich, Germany) supplemented with $1 \%$ antibiotic-antimycotic solution (Biosera, France) and 5\% fetal bovine serum (FBS; Sigma-Aldrich, Germany) in Nunc 4-well culture plates (Thermo Fisher Scientific, USA) (1 ovary/1 well/500 $\mu \mathrm{l}$ medium) in $5 \% \mathrm{CO}_{2}$ incubator at $37^{\circ} \mathrm{C}$. The ovaries of each experimental group were cultured in the absence or presence of hormones [recombinant follicle-stimulating hormone ( $\mathrm{rFSH}$, Puregon, N.V. Organon, Netherlands, 100 ng/ml); recombinant luteinizing hormone $(\mathrm{rLH}$, Luveris, Merck, Netherlands, $100 \mathrm{ng} / \mathrm{ml}$ ); and recombinant human insulin-like growth factor-1 (IGF-1, PeproTech EC, Ltd., UK, $100 \mathrm{ng} / \mathrm{ml}$ )] for 6 days. On day 1, 3, and 6 , the culture media were collected for hormone analysis; on day 1 and 3 , the collected culture media were replaced with fresh ones.

\section{Experiment 2: $\mathrm{TiO}_{2} \mathrm{NP}$ exposure.}

Preparation and characterization of $\mathrm{TiO}_{2} \mathrm{NP}$ suspension. Titanium (IV) oxide nanopowder [<100 nm (BET), 99.9\% metal basis, Sigma-Aldrich, Cat. No. 634662, CAS 13463-67-7, FW 789.9] was suspended in the physiological solution containing $10 \%(\mathrm{v} / \mathrm{v})$ of rat serum (Sigma-Aldrich, Germany; $\mathrm{pH}=7.5)$ at concentration $59.22 \mathrm{mg} / 10 \mathrm{ml}$, sonicated (Sonopuls, Bandelin electronic, Germany) for $15 \mathrm{~min}$ at $150 \mathrm{~W}$. Fresh suspension was prepared daily and immediately before administration, it was vortexed at the highest speed for $1 \mathrm{~min}$.

DLS was used to measure average particle size, hydrodynamic size distribution, and degree of aggregation and zeta potential of $\mathrm{TiO}_{2}$ NPs in $10 \%$ rat serum physiological solution at concentration 1 $\mathrm{mg} / \mathrm{ml}$. DLS measurements were performed with a Malvern Instrument Zetasizer Nano equipped with a He-Ne laser $(\lambda=633 \mathrm{~nm}$, max $5 \mathrm{~mW})$ and operated at a scattering angle of $173^{\circ}$. The mean hydrodynamic diameter was calculated from the autocorrelation function of the intensity of light scattered from the particles. The temperature of standard measurements was set to $25^{\circ} \mathrm{C}$ or $37^{\circ} \mathrm{C}$.

Analysis of $\mathrm{TiO}_{2} \mathrm{NPs}$ solution by DLS showed a polydisperse suspension of one population of particles with $308.6 \pm 2.5 \mathrm{~nm}(100 \%)$ and average size of $244.4 \pm 0.9 \mathrm{~nm}$ measured at $25^{\circ} \mathrm{C}$. DLS analysis of $\mathrm{TiO}_{2} \mathrm{NPs}$ solution performed at $37^{\circ} \mathrm{C}$ showed one population of particles with $279.39 \pm 17.1 \mathrm{~nm}$ (100\%) and average diameter of $256.8 \pm 7.9 \mathrm{~nm}$. Zeta potential was $-13.81 \pm 0.35 \mathrm{mV}$ and $-13.18 \pm 0.38 \mathrm{mV}$ measured at $25^{\circ} \mathrm{C}$ and $37^{\circ} \mathrm{C}$, respectively. Polydispersity index (PDI) of $\mathrm{TiO}_{2}$ NPs solution was 
$0.205 \pm 0.010$ and $0.223 \pm 0.032$ measured at $25^{\circ} \mathrm{C}$ and $37^{\circ} \mathrm{C}$, respectively.

Choice of doses and study design. The study was carried out on one generation of the neonatal rat model (Newbold et al. 2007; Picut et al. 2015). Anticipating that human exposure to $\mathrm{TiO}_{2}$ is rather low, the neonatal female rats were short-time exposed to two low doses (1\% and $10 \%)$ derived from the estimated value of $\mathrm{LD}_{50}$ of $\mathrm{TiO}_{2} \mathrm{NPs}$ (administered by the single intravenous, i.v., injection) calculated as $59.22 \mathrm{mg} / \mathrm{kg}$ b.w., with the confidence interval from 55 to $70 \mathrm{mg} / \mathrm{kg}$ (Sebekova et al. 2014). Two doses of $\mathrm{TiO}_{2} \mathrm{NPs}$ were used according to the Endocrine disruptor screening program test guidelines (EDSPTG) OPPTS 890.1450 and the study by Newbold et al. (2007) as the present experiment is an integral part of reproductive and neuroendocrine toxicity study in female rats neonatally exposed to $\mathrm{TiO}_{2} \mathrm{NPs}$.

In the present study, 32 neonatal female rats were used. Parent specific pathogen free Wistar rats were obtained from Breeding Facility VELAZ, Prague (Czech Republic). Newborn female rats were i.p. injected daily with two different doses of $\mathrm{TiO}_{2}$ NPs: $1 \% \mathrm{LD}_{50} \mathrm{TiO}_{2}=59.2 \mu \mathrm{g} / \mathrm{kg}$ b.w. $(\mathrm{n}=10)$ and $10 \% \mathrm{LD}_{50}$ $\mathrm{TiO}_{2}=592 \mu \mathrm{g} / \mathrm{kg}$ b.w. $(\mathrm{n}=11)$ in $10 \%(\mathrm{v} / \mathrm{v})$ rat serum physiological solution or vehicle $(10 \%$ rat serum physiological solution, $\mathrm{v} / \mathrm{v}, \mathrm{n}=11$ ) from the PND4 to the PND7 in the dose volume of $10 \mathrm{ml} / \mathrm{kg}$ b.w. Neonatally exposed infantile female rats were sacrificed on the PND15 by decapitation under ketamine (Narketan, Vetoquinol Ltd., Czech Republic)/xyla- sine (Xylariem, Riemser Arzneimittel AG, Germany) anesthesia $(60 / 10 \mathrm{mg} / \mathrm{kg}$ of b.w.) and the ovaries from each experimental group were collected for ex vivo ovary culture.

Ex vivo ovary culture. At PND15, the collected ovaries were cut into 2 pieces and cultured in vitro in M199 (with Earle salt, $1 \mathrm{mM} \mathrm{L}$-glutamine, and HEPES; Sigma-Aldrich, Germany) supplemented with $1 \%$ antibiotic-antimycotic solution and 5\% FBS in 24-well plates (TPP, Switzerland) (half ovary/ 1 well $/ 500 \mu \mathrm{l}$ medium) in $5 \% \mathrm{CO}_{2}$ incubator at $37^{\circ} \mathrm{C}$. The ovaries were cultured in the absence or presence of hormones (rFSH, $100 \mathrm{ng} / \mathrm{ml}$; IGF-1, $100 \mathrm{ng} / \mathrm{ml}$ ) for 6 days. Every other day (day 2, 4, 6), culture media were collected for hormone analysis and on day 2 and 4 replaced with fresh medium.

Study approval. The animal part of both studies/ experiments was approved by State Veterinary and Food Administration of SR according to Government order No 39/2007 Z.z. and No. 377/2012 Z.z. laying down requirements for protection of animals used for experimental purposes or other scientific purposes. All procedures were performed according to Standard Operating Procedures (SOPs) established at Department of Toxicology, Slovak Medical University in Bratislava in compliance with the Good Laboratory Practice (GLP) principles.

Steroid hormone analysis. Steroid hormone levels in the culture media from both experiments were analyzed by specific radioimmunoassay (RIA) kits or enzyme-linked immunosorbent assay (ELISA)

Table 1

Body and ovary weights of female Wistar rats neonatally exposed to PEG- $b$-PLA nanoparticles on the day of ovariectomy.

\begin{tabular}{lccc}
\hline Treatment groups & Control & PEG- $\boldsymbol{b}$-PLA 20 & PEG- $\boldsymbol{b}$-PLA 40 \\
\hline Dose/Day (mg/kg b.w.) & 0 & 20 & 40 \\
No of animals & 12 & 13 & 9 \\
Body weight (g) & $249.49 \pm 4.64$ & $251.88 \pm 5.03$ & $253.77 \pm 2.33$ \\
Ovary weight & & & \\
$(\mathrm{mg})$ & $82.59 \pm 2.75$ & $86.85 \pm 3.54$ & $80.48 \pm 3.64$ \\
$(\mathrm{mg} \%)$ & $33.10 \pm 0.90$ & $34.53 \pm 1.38$ & $31.67 \pm 1.29$ \\
Left ovary & & & \\
$(\mathrm{mg})$ & $42.37 \pm 1.62$ & $42.45 \pm 2.04$ & $39.97 \pm 1.94$ \\
$(\mathrm{mg} \%)$ & $17.01 \pm 0.64$ & $16.91 \pm 0.83$ & $15.73 \pm 0.70$ \\
Right ovary & & & \\
$(\mathrm{mg})$ & $40.23 \pm 1.62$ & $44.39 \pm 2.20$ & $40.51 \pm 2.19$ \\
$(\mathrm{mg} \%)$ & $16.09 \pm 0.49$ & $17.62 \pm 0.82$ & $15.94 \pm 0.79$ \\
\hline
\end{tabular}

Data are presented as mean \pm SEM. $\mathrm{mg} \%$ - organ weight $(\mathrm{mg}) /$ body weight $(\mathrm{g}) \times 100$.

Abbreviations: b.w. - body weight. 
kit. Progesterone concentrations in the culture media were measured by Progesterone [125I] RIA kit (RK-460M, IZOTOP, Institute of Izotopes Ltd., Budapest, Hungary). The sensitivity of the assay was $0.44 \pm 0.12 \mathrm{nM}$, intra-assay and inter-assay CVs were $10.2 \%$ and CV 11.8\%, respectively. Estradiol concentrations in the culture media were measured by ImmuChem Double Antibody 17 $\beta$-Estradiol [125I] RIA kit (07-138102, MP Biomedicals Germany $\mathrm{GmbH}$, Geramny) or by ELISA kit for estradiol (CEA461Ge, Cloud-Clone Corp., USA). The sensitivity of estradiol RIA kit was $10 \mathrm{pg} / \mathrm{ml}$, intra-assay and inter-assay CVs were $10.7 \%$ and CV $11.9 \%$, respectively. The sensitivity of ELISA kit for estradiol was $4.45 \mathrm{pg} / \mathrm{ml}$, intra-assay and inter-assay CVs were $<10 \%$ and $<12 \%$, respectively.

Statistical analysis. The data are expressed as the mean \pm standard error of the mean (SEM). Number of samples are indicated in Figure legend. Statistical analysis was performed with a One-way Analysis of Variance (ANOVA) followed by Tukey-Kramer or Bonferroni multiple comparisons test. Values of $\mathrm{p}<0.05$ were considered significant. Sigma Plot 11.0 software (Systat Software, GmbH, Erkhart, Germany) was used for statistical analysis.

\section{Results}

\section{Experiment 1: PEG- $b$-PLA NP exposure.}

The neonatal exposure (PND4-7) to polymeric PEG- $b$-PLA NPs, at both concentrations tested (20 and $40 \mathrm{mg} / \mathrm{kg}$ b.w.), did not significantly affect the body and ovary weights in adult (PND73) female rats compared with control group (Table 1).

Basal progesterone secretion by ovaries of both PEG- $b$-PLA-treated groups (PEG- $b$-PLA20 and PEG- $b$-PLA40) cultured for 3 days was significantly decreased $(\mathrm{p}<0.01)$ compared with controls (Figure 1B). After 1 day and 6 days of the culture, no effects on basal progesterone secretion by ovaries of rats neonatally exposed to PEG- $b$-PLA NPs were observed compared with controls (Figures 1A,C). The presence of gonadotropins (FSH, LH) and IGF-1 in the culture medium induced a significant stimulation $(\mathrm{p}<0.001)$ of progesterone production by ovaries of control rats at all time intervals compared with basal levels (Figures 1A,B,C). Neonatal treatment of rats with lower dose of PEG- $b$-PLA NPs (PEG- $b$-PLA20) did not alter the stimulatory effect of hormones on progesterone secretion (Figures 1A,B,C). Stimulated progesterone production by ovaries of PEG- $b$-PLA40treated group was significantly decreased $(\mathrm{p}<0.05)$ after 3 days of culture compared with controls (Figure 1B).
Figure 2 shows time-course (1-6 days) of basal and stimulated progesterone secretion by ovaries of control and PEG- $b$-PLA-treated groups. Timedependent significant reduction of basal progesterone production was found in both PEG- $b$-PLA-treated

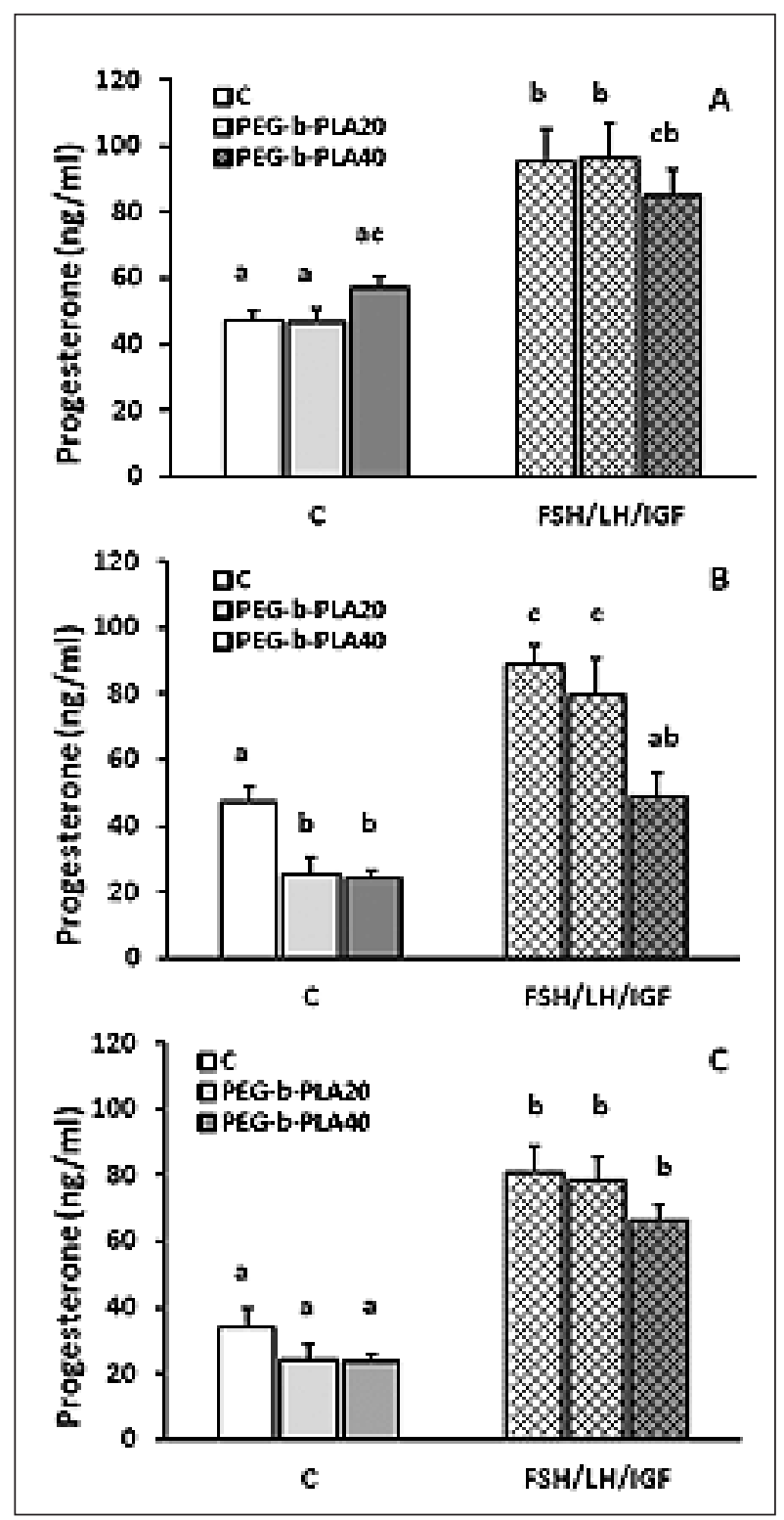

Figure 1. Basal (C) and stimulated (FSH/LH/IGF-1) progesterone secretion by ex vivo cultured ovaries from adult (PND73) rats neonatally exposed to vehicle $(\mathrm{C})$ or two doses of polymeric PEG- $b$-PLA NPs (20 and $40 \mathrm{mg} / \mathrm{kg}$ body weight) (PEG$b$-PLA20, PEG- $b$-PLA40) after 1 day (A), 3 days (B) and 6 days (C) of the culture. Data are presented as mean \pm SEM, $n=5-6$. Data were analyzed by ANOVA followed by Tukey-Kramer post-test, values of $\mathrm{p}<0.05$ were considered significant. Different superscripts indicate significant differences between groups. 
groups (PEG- $b$-PLA20, $\mathrm{p}<0.05 ; \quad$ PEG- $b$-PLA40, $\mathrm{p}<0.001$ ) (Figure 2A). Slight time-dependent decrease of stimulated progesterone secretion was observed at all experimental groups, with a significant inhibition $(\mathrm{p}<0.01)$ in PEG- $b$-PLA20-treated group after 3 days of culture (Figure 2B).

Basal estradiol secretion by ovaries of both PEG$b$-PLA-treated groups (PEG- $b$-PLA20 and PEG- $b$ PLA40) cultured for 1 day was significantly lower $(\mathrm{p}<0.001)$ than in controls (Figure 3$)$. With the presence of gonadotropins (FSH, LH) and IGF-1 in the culture medium, estradiol production significantly increased $(\mathrm{p}<0.001)$ and did not differ between control and PEG- $b$-PLA-treated groups (Figure 3).

Experiment 2: $\mathrm{TiO}_{2} \mathrm{NP}$ exposure.

Terminal body and ovary weights of infantile (PND15) female rats neonatally exposed to two doses

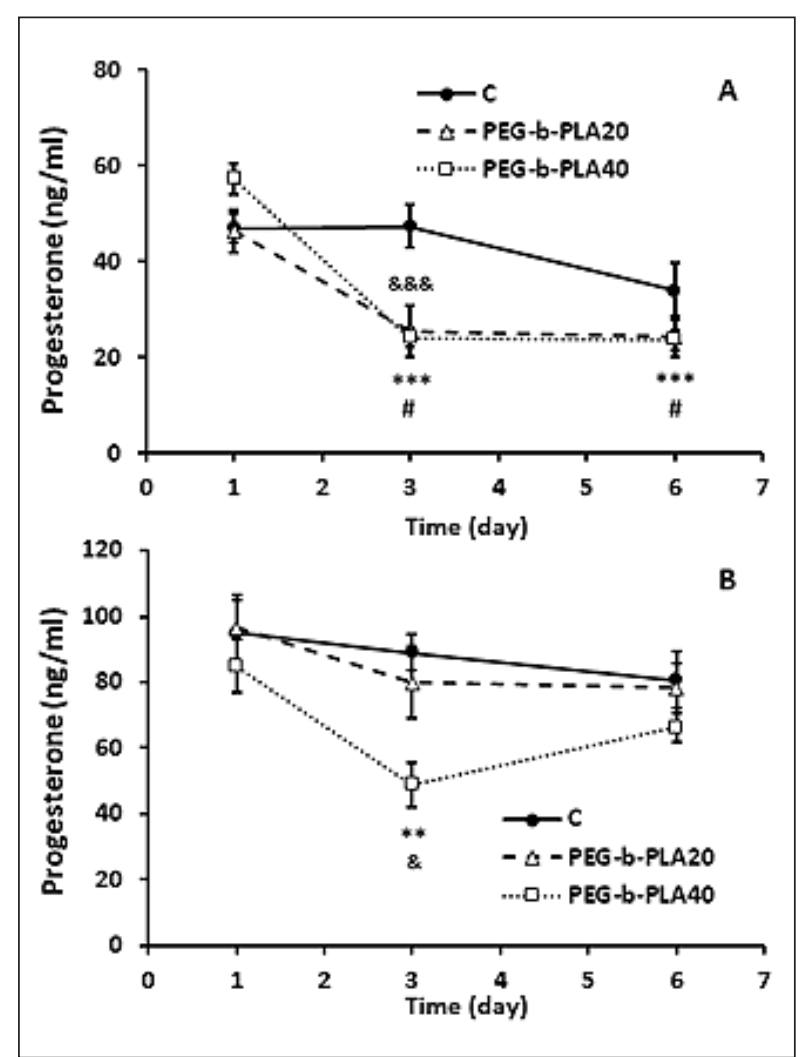

Figure 2. Time-course of basal (A) and FSH/LH/IGF-1-stimulated (B) progesterone secretion by ex vivo cultured ovaries from adult (PND73) rats neonatally exposed to vehicle (C) or two doses of polymeric PEG- $b$-PLA NPs (20 and $40 \mathrm{mg}$ / kg body weight) (PEG- $b$-PLA20, PEG- $b$-PLA40). Data are presented as mean \pm SEM, $n=5-6$. Data were analyzed by ANOVA followed by Bonferroni multiple comparisons test. ${ }^{* *} \mathrm{p}<0.001$ vs. PEG-bPLA40 on day $1 ;{ }^{*} \mathrm{p}<0.05$ vs. PEG-bPLA2 0 on day 1 ; ${ }^{\& \& \&} \mathrm{p}<0.001$ vs. $\mathrm{C}$ on day 3 . of $\mathrm{TiO}_{2} \mathrm{NPs}\left(1 \% \mathrm{LD} 50 \mathrm{TiO}_{2}\right.$ and 10\%LD50 $\mathrm{TiO}_{2}$ ) were not significantly changed compared with control animals (Table 2).

After ex vivo culture of rat ovaries collected on PND15, basal progesterone levels measured in culture media at all time intervals (2, 4, and 6 days) were not significantly altered by the action of both doses of $\mathrm{TiO}_{2}$ NPs $\left(1 \% \mathrm{LD} 50 \mathrm{TiO}_{2}\right.$ and $10 \% \mathrm{LD} 50 \mathrm{TiO}_{2}$ ) compared with controls (Figures 4A,B,C). The presence of FSH and IGF-1 in the culture medium induced a significant stimulation $(\mathrm{p}<0.01)$ of progesterone production by ovaries of control rats after 2 and 4 days of culture compared with basal progesterone levels (Figures 4A,B,C). After 6 days of culture, no increase in progesterone production by FSH/IGF-1 stimulation was found in control group compared with basal levels (Figure 4C). The ovaries from rats neonatally exposed to both doses of $\mathrm{TiO}_{2} \mathrm{NPs}\left(1 \% \mathrm{LD} 50 \mathrm{TiO}_{2}\right.$ and $10 \% \mathrm{LD} 50 \mathrm{TiO}_{2}$ ) failed to respond to FSH/IGF stimulation in progesterone secretion at all time intervals (Figures $4 A, B, C)$. Significant decrease $(p<0.05)$ in stimulated progesterone production was found in $1 \% \mathrm{LD} 50 \mathrm{TiO}_{2}$-treated group after 4 days of culture compared with controls (Figure 4B).

Figure 5 shows time-course (2-6 days) of basal and stimulated progesterone secretion by ovaries of control and $\mathrm{TiO}_{2}$ NP-treated groups. No significant changes in basal progesterone secretion with time in control and both $\mathrm{TiO}_{2}$ NP-treated groups were found

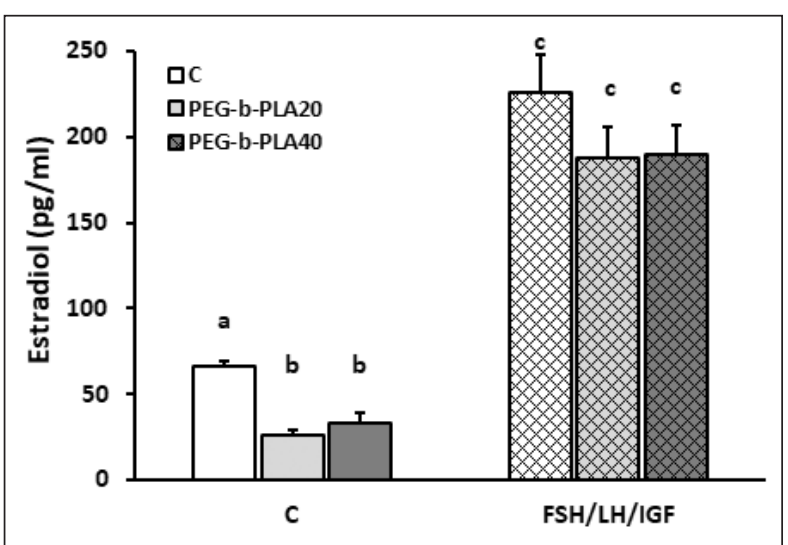

Figure 3. Basal (C) and stimulated (FSH/LH/IGF-1) estradiol secretion by ex vivo cultured ovaries from adult (PND73) rats neonatally exposed to vehicle (C) or two doses of polymeric PEG- $b$-PLA NPs (20 and $40 \mathrm{mg} / \mathrm{kg}$ body weight) (PEG- $b$ PLA20, PEG- $b$-PLA40) after 1 day of the culture. Data are presented as mean \pm SEM, $n=5-6$. Data were analyzed by ANOVA followed by Tukey-Kramer post-test, values of $p<0.05$ were considered significant. Different superscripts indicate significant differences between groups. 
(Figure 5A). Stimulated progesterone production by ovaries of control animals was significantly decreased $(\mathrm{p}<0.01)$ after 6 days of culture compared with levels after 2 days of culture (Figure 5B). Significant reduction in stimulated progesterone secretion by ovaries of $1 \% \mathrm{LD} 50 \mathrm{TiO}_{2}$-treated rats was demonstrated after 4 days of culture compared with controls (Figure 5B).

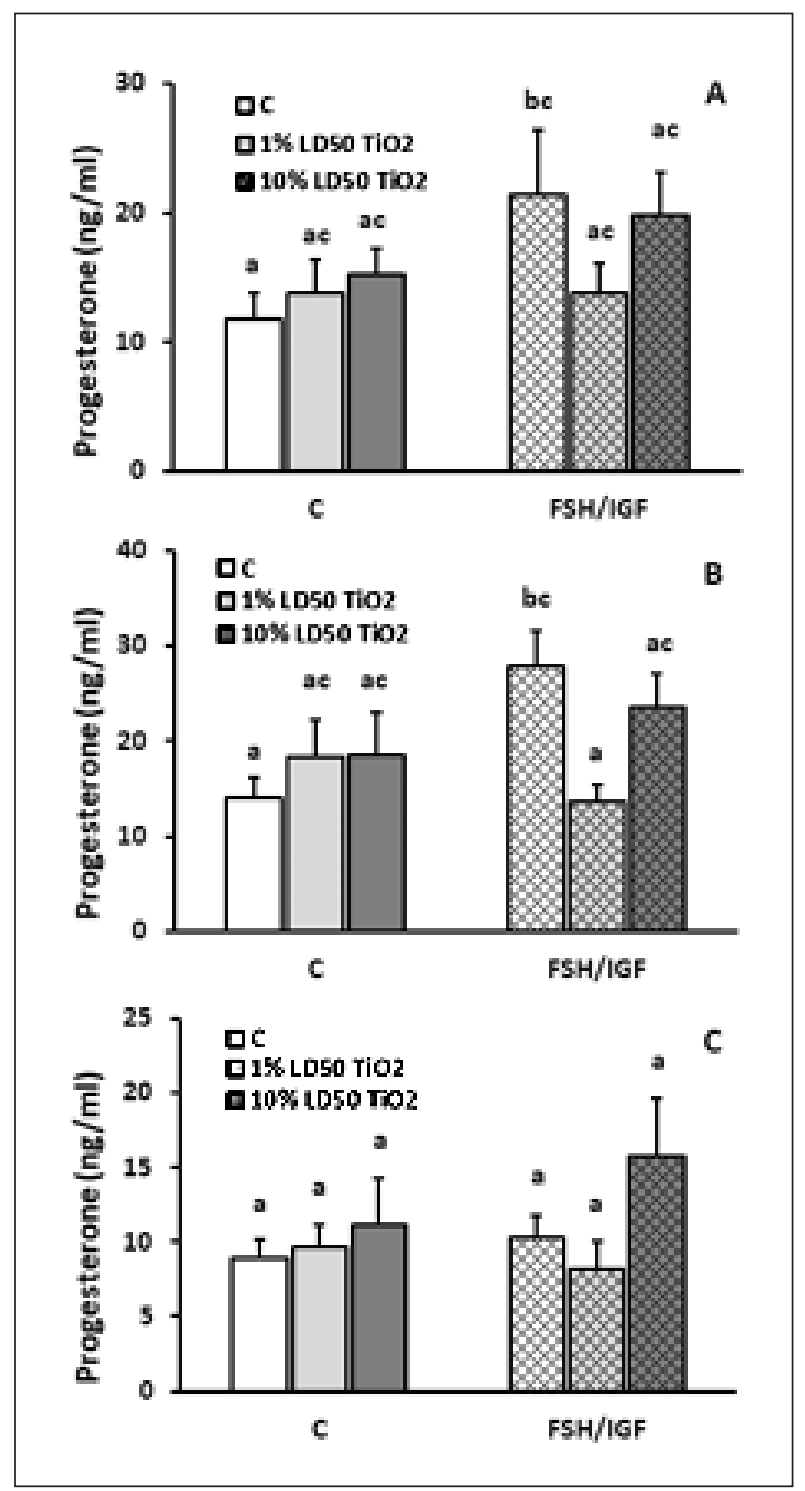

Figure 4. Basal (C) and stimulated (FSH/LH/IGF-1) progesterone secretion by ex vivo cultured ovaries from infantile (PND15) rats neonatally exposed to vehicle (C) or two doses of titanium dioxide $\left(\mathrm{TiO}_{2}\right)$ NPs $\left(1 \% \mathrm{LD}_{0} \mathrm{TiO}_{2}=59.2 \mu \mathrm{g} / \mathrm{kg}\right.$ b.w. and $10 \% \mathrm{LD}_{0} \mathrm{TiO}_{2}=592 \mu \mathrm{g} / \mathrm{kg}$ b.w. $)\left(1 \% \mathrm{LD}_{0} \mathrm{TiO}_{2}, 10 \%\right.$ LD50 $\mathrm{TiO}_{2}$ ) after 2 days (A), 4 days (B) and 6 days (C) of the culture. Data are presented as mean $\pm S E M, n=10-11$. Data were analyzed by ANOVA followed by Tukey-Kramer post-test, values of $\mathrm{p}<0.05$ were considered significant. Different superscripts indicate significant differences between groups.
Basal estradiol secretion by rat ovaries did not differ between control and $\mathrm{TiO}_{2}$ NP-treated groups after 2 and 4 days of culture (Figures 6A,B). After 6 days of culture, basal estradiol levels were significantly decreased $(\mathrm{p}<0.05)$ in $10 \% \mathrm{LD} 50 \mathrm{TiO}_{2}$ NP-treated group compared with controls (Figure 6C). The presence of FSH and IGF-1 in the culture medium did not induce stimulation of estradiol production in all experimental groups in all time intervals compared with basal levels (Figures 6A,B,C).

\section{Discussion}

The present experiments are integral parts of reproductive and neuroendocrine toxicity studies in female rats in two different life stages (infantile and adult) neonatally exposed to PEG- $b$-PLA and $\mathrm{TiO}_{2}$ NPs. The investigated reproductive and neuroendocrine endpoints obtained from these studies are (Roll-

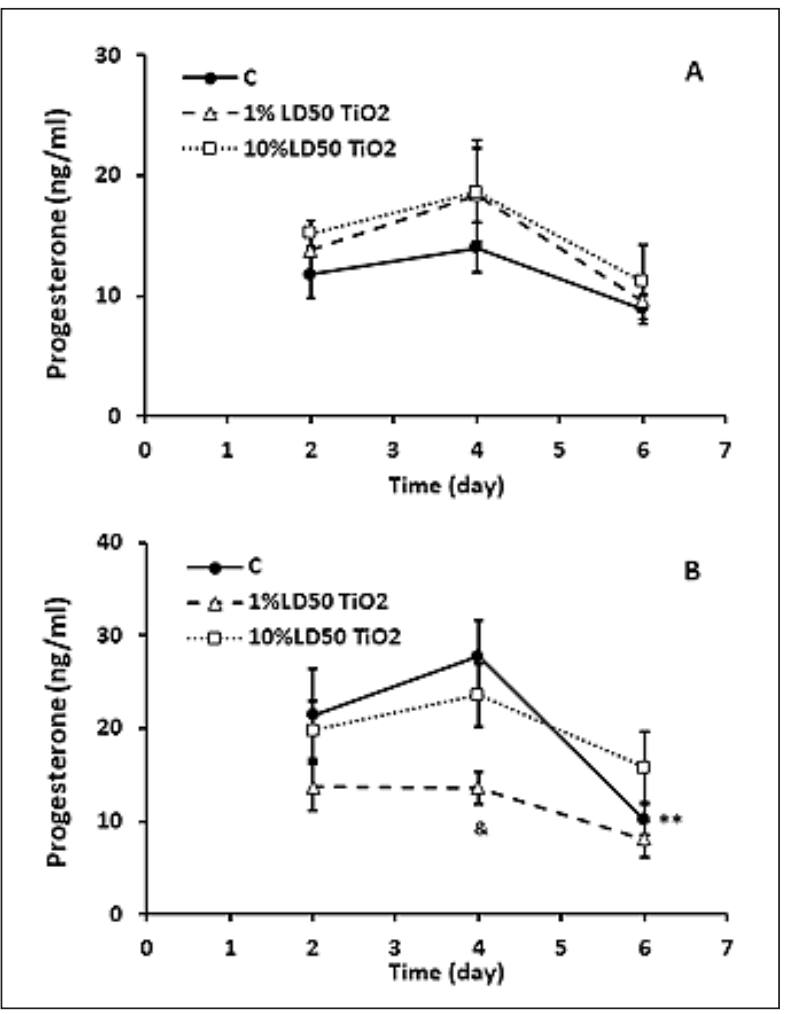

Figure 5. Time-course of basal (A) and FSH/LH/IGF-1-stimulated (B) progesterone secretion by ex vivo cultured ovaries from infantile (PND15) rats neonatally exposed to vehicle (C) or two doses of titanium dioxide $\left(\mathrm{TiO}_{2}\right) \mathrm{NPs}\left(1 \% \mathrm{LD} 50 \mathrm{TiO}_{2}=59.2 \mu \mathrm{g} /\right.$ kg b.w. and $10 \% \mathrm{LD}_{0} \mathrm{TiO}_{2}=592 \mu \mathrm{g} / \mathrm{kg}$ b.w. $)\left(1 \% \mathrm{LD}^{2} \mathrm{TiO}_{2}\right.$, $10 \% \mathrm{LD}_{0} \mathrm{TiO}_{2}$ ). Data are presented as mean \pm SEM, $\mathrm{n}=10-11$. Data were analyzed by ANOVA followed by Bonferroni multiple comparisons test. ${ }^{* *} \mathrm{p}<0.01$ vs. $\mathrm{C}$ on day $6 ;{ }^{8} \mathrm{p}<0.051$ vs. $1 \% \mathrm{LD}_{0} \mathrm{TiO}_{2}$ on day 4 . 
Table 2

Body and ovary weights of female Wistar rats neonatally exposed to titanium dioxide $\left(\mathrm{TiO}_{2}\right)$ nanoparticles on the day of necropsy.

\begin{tabular}{lccc}
\hline Treatment groups & Control & $\begin{array}{c}\text { TiO2 } \\
(\mathbf{1 \% L D 5 0 )}\end{array}$ & $\begin{array}{c}\text { TiO2 } \\
(\mathbf{1 0 \% L D 5 0})\end{array}$ \\
\hline $\begin{array}{l}\text { Dose/Day }(\mu \mathrm{g} / \mathrm{kg} \text { b.w.) } \\
\text { No of animals }\end{array}$ & 0 & 59.22 & 592.2 \\
\hline Body weight $(\mathrm{g})$ & 11 & 10 & 11 \\
Ovary weight & $40.37 \pm 1.51$ & $36.03 \pm 1.92$ & $39.88 \pm 0.88$ \\
(mg) & & & \\
(mg\%) & $5.74 \pm 0.68$ & $6.07 \pm 0.86$ & $6.35 \pm 0.55$ \\
Left ovary & $14.04 \pm 1.37$ & $16.59 \pm 2.10$ & $15.87 \pm 1.32$ \\
(mg) & & & \\
(mg\%) & $3.25 \pm 0.30$ & $2.76 \pm 0.48$ & $3.15 \pm 0.27$ \\
Right ovary & $7.91 \pm 0.46$ & $7.72 \pm 1.25$ & $7.87 \pm 0.64$ \\
(mg) & & & \\
(mg\%) & $2.48 \pm 0.56$ & $3.31 \pm 0.52$ & $3.19 \pm 0.32$ \\
\hline
\end{tabular}

Data are presented as mean \pm SEM. $\mathrm{mg} \%$ - organ weight $(\mathrm{mg}) /$ body weight $(\mathrm{g}) \times 100$.

Abbreviations: b.w. - body weight.

erova et al. 2015a; Scsukova et al. 2015; Dvorakova et al. 2017; Scsukova et al. 2017) or will be the subject of other articles. In the present paper, we present a part of these studies investigating possible alterations of ovarian steroidogenic capacity in adult (PND73) and infantile (PND15) rats neonatally (PND4-7) exposed to polymeric PEG- $b$-PLA and $\mathrm{TiO}_{2}$ NPs, respectively, using ex vivo ovary cultures.

We observed significant decrease in basal progesterone and estradiol secretion by ovaries from PEG$b$-PLA-treated rats after 3 days and 1 day of ex vivo ovary culture, respectively, compared with control group. FSH/LH/IGF-1-stimulated progesterone levels were significantly decreased in PEG- $b$-PLA40-treated group after 3 days of culture compared with controls. FSH/LH/IGF-1-stimulated estradiol production was not affected in PEG- $b$-PLA-treated groups. To our best knowledge, the adverse effects of polymeric NPs have not been extensively studied and only few studies have focused on their effects on reproduc-

Figure 6. Basal (C) and stimulated (FSH/LH/IGF-1) estradiol secretion by ex vivo cultured ovaries from infantile (PND15) rats neonatally exposed to vehicle $(\mathrm{C})$ or two doses of titanium dioxide $\left(\mathrm{TiO}_{2}\right)$ NPs $\left(1 \% \mathrm{LD}_{0} \mathrm{TiO}_{2}=59.2 \mu \mathrm{g} / \mathrm{kg}\right.$ b.w. and $10 \%$ LD50 $\mathrm{TiO}_{2}=592 \mu \mathrm{g} / \mathrm{kg}$ b.w. $)\left(1 \% \mathrm{LD}_{0} \mathrm{TiO}_{2}, 10 \% \mathrm{LD} 50 \mathrm{TiO}_{2}\right)$ after 2 days (A), 4 days (B) and 6 days (C) of the culture. Data are presented as mean \pm SEM, $n=10-11$. Data were analyzed by ANOVA followed by Tukey-Kramer post-test, values of $\mathrm{p}<0.05$ were considered significant. Different superscripts indicate significant differences between groups.
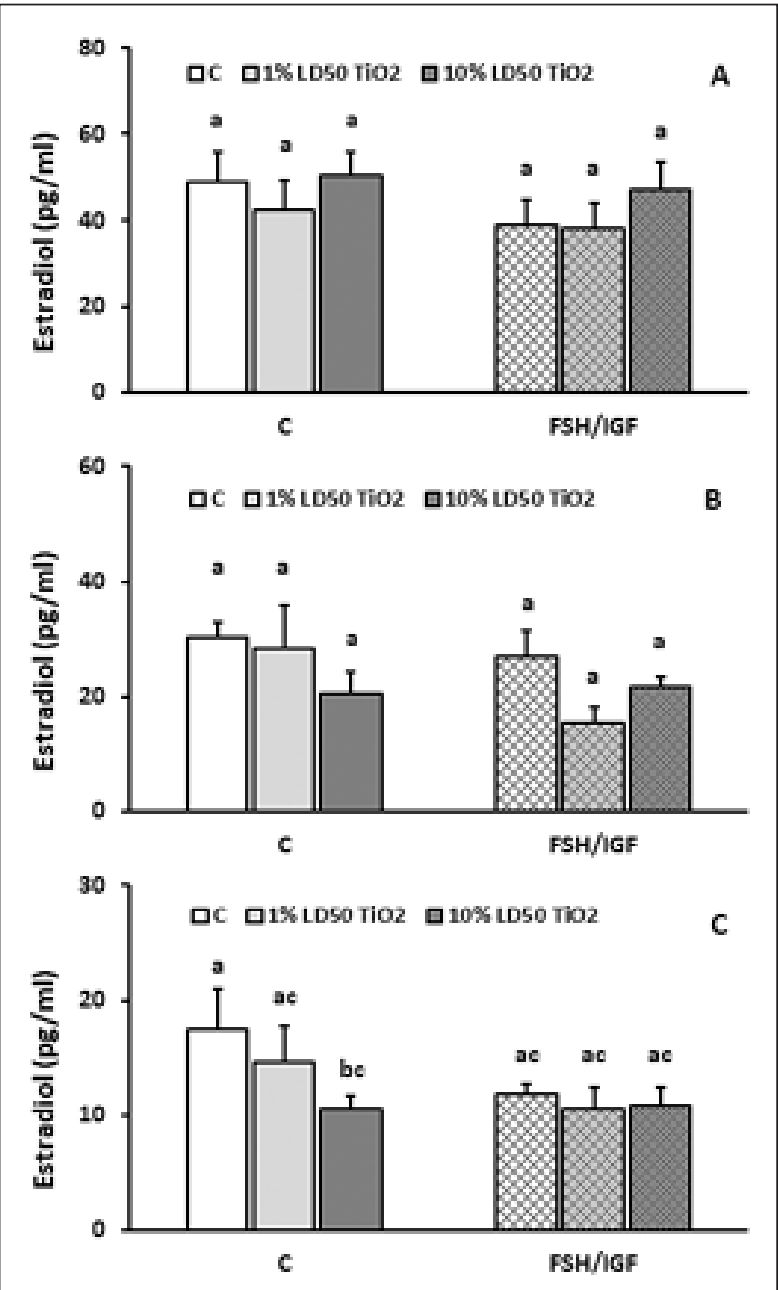
tive system to date. Previously, we have shown that neonatal exposure to PEG- $b$-PLA NPs $(20,40 \mathrm{mg} / \mathrm{kg}$ b.w.) may induce delayed adverse effects on hypothalamic-pituitary-ovarian axis development (accelerated onset of puberty) and function [reduced number of regular estrous cycles, altered course of hypothalamic gonadotropin-releasing hormone (GnRH)stimulated LH secretion, and increased progesterone serum levels] in 6-month-old female rats (Rollerova et al. 2015a). In addition, neonatal treatment with a lower dose of PEG-b-PLA NP (20 mg/kg b.w.) significantly increased basal and LHRH-induced in vitro LH release from anterior pituitary cells of infantile and adult female rats (Scsukova et al. 2015). Investigating in vitro effects of polymeric PEG- $b$-PLA NPs on ovarian steroidogenesis using primary culture of porcine granulosa cells (GCs), we demonstrated that treatment of GCs with PEG- $b$-PLA NPs induced a significant decrease in basal as well as FSH-stimulated progesterone secretion above the concentration of 20 and $4 \mu \mathrm{g} / \mathrm{ml}$, respectively. Moreover, PEG$b$-PLA NPs reduced forskolin-stimulated, but not cAMP-stimulated progesterone production by GCs. A dose-dependent inhibition of androstenedionestimulated estradiol release by GCs was found by the action of PEG- $b$-PLA NPs (Scsukova et al. 2017).

In summary, our results of in vivo and in vitro studies indicate that polymeric PEG- $b$-PLA NPs may induce alteration of ovarian steroidogenesis and reproductive functions. However, the molecular mechanisms of the action of PEG- $b$-PLA NPs on female reproductive system are still unknown. The adverse effects of polymeric NPs on reproductive system have not been extensively studied and only few studies have focused on their effects to date. Xiao et al. (2011) investigating the cellular uptake mechanisms of PEG- $b$-PLA micelles demonstrated that they firstly interacted with cell membrane, induced cell membrane depolarization and enhance membrane microviscosity in a dose-dependent manner. Previously, we have reported that cell membrane microenvironment play an essential role in regulation of structure-function relationship of gonadotropin receptors (Kolena et al. 1995). The obtained results might indicate that understanding of interaction of NPs with cell membranes and their internalization into the cells is important in determining their diverse biological responses.

The ovaries collected from infantile rats secreted less basal progesterone and estradiol into the culture media compared with ovaries form adult rats. Moreover, ovaries from infantile rats did not respond to FSH/IGF-1 stimulation in estradiol secre- tion. Neonatal exposure of female rats to $\mathrm{TiO}_{2} \mathrm{NPs}$ (1\%LD50 $\mathrm{TiO}_{2}$ and $10 \% \mathrm{LD} 50 \mathrm{TiO}_{2}$ ) did not affect basal progesterone and estradiol secretion by ovaries after ex vivo culture compared with control group. The ovaries from rats neonatally exposed to both doses of $\mathrm{TiO}_{2}$ NPs failed to respond to FSH/IGF stimulation in progesterone secretion at all time intervals examined.

Many in vivo studies have clearly shown that $\mathrm{TiO}_{2}$ NPs administered in different ways can migrate through systemic circulation and biological barriers to different organs, including ovary, accumulate in them, and finally cause serious injury (Iavicoli et al. 2012; Geraets et al. 2014; Tassinari et al. 2014; Rollerova et al. 2015b). Gao et al. (2012) demonstrated that chronic $\mathrm{TiO}_{2}$ NPs exposure significantly decreased serum levels of progesterone, testosterone and gonadotropins, and increased concentration of estradiol, resulting in increased atresia of the primary and secondary follicle development and reduced fertility in female mice. Short-term exposure to anatase $\mathrm{TiO}_{2}$ NPs modulated serum concentrations of sex steroid hormones in male and female rodents (Tassinari et al. 2014). $\mathrm{TiO}_{2}$ NPs reduced the developmental success of CD-1 mice after a single dose to dams, resulting in a statistically significant increase in fetal deformities and mortality (Philbrook et al. 2011). Zhao et al. (2013) suggested that fertility reduction and ovary injury of mice following exposure toTiO $\mathrm{NPs}_{2} \mathrm{Nay}$ be associated with alteration of inflammation-related or follicular atresia-related cytokine expressions. Numerous in vitro studies indicate that $\mathrm{TiO}_{2} \mathrm{NPs}$ may pose toxic effects by inducing oxidative stress, cytotoxicity, genotoxicity, inflammation and cell apoptosis (Iavicoli et al. 2011; Arora et al. 2012; Shi et al. 2013; Zhang et al. 2015).

The obtained results indicate that neonatal exposure to NPs in female rats may alter ovarian steroidogenic output (steroid hormone secretion) and thereby might subsequently induce perturbation of mammalian reproductive functions. The molecular mechanisms of adverse effects of NPs on ovarian functions, including induction of oxidative stress and inflammation, remain unclear and should be further elucidated.

\section{Acknowledgements}

The authors would like to thank Dagmar Bilanicova, PhD. (Qi Technologies s.r.l., Pomezia, Italy) and Josef Kollar, PhD. (Polymer Institute, Slovak Academy of Sciences, Bratislava, Slovakia) for characterization of PEG- $b$-PLA and $\mathrm{TiO}_{2} \mathrm{NP}$ suspensions, respectively. This work was supported by the 
Slovak Research and Development Agency under the contracts No. APVV-0404-11 and No. APVV-150296; and VEGA Grant 2/0187/17. In addition, it was supported by the realization of the project "Center of Excellence of Environmental Health" ITMS No. 26240120033, based on supporting operational research and development program financed from the European Regional Development Fund.

\section{References}

Alaee S, Ilani M. Effect of titanium dioxide nanoparticles on male and female reproductive systems. J Adv Med Sci Appl Technol 3, 3-8, 2017.

Arora S, Rajwade JM, Paknikar KM. Nanotoxicology and in vitro studies: the need of the hour. Toxicol Appl Pharmacol 258, 151-165, 2012.

Casalini T, Rossi F, Castrovinci A, Perale G. A perspective on polylactic acid-based polymers use for nanoparticles synthesis and applications. Front Bioeng Biotechnol 7, 259, 2019.

Das J, Choi YJ, Song H, Kim JH. Potential toxicity of engineered nanoparticles in mammalian germ cells and developing embryos: Treatment strategies and anticipated applications of nanoparticles in gene delivery. Hum Reprod Update 22, 588-619, 2016.

Cho H, Gao J, Kwon GS. PEG-b-PLA micelles and PLGA-b-PEG-b-PLGA sol-gels for drug delivery. J Control Release 240, 191-201, 2016.

De Jong WH, Borm PJ. Drug delivery and nanoparticles: applications and hazards. Int J Nanomedicine 3, 133-149, 2008.

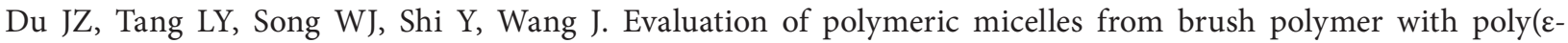
caprolactone)-b-poly(ethyleneglycol) side chains as drug carrier. Biomacromolecules 10, 2169-2174, 2009.

Dvorakova M, Rollerova E, Scsukova S, Bujnakova Mlynarcikova A, Laubertova L, Zitnanova I. Effect of neonatal exposure to poly(ethylene glycol)-block-poly(lactic acid) nanoparticles on oxidative state in infantile and adult female rats. Oxid Med Cell Longev 2017, 7430435, 2017.

Ferrandina G, Corrado G, Licameli A, Lorusso D, Fuoco G, Pisconti S, Scambia G. Pegylated liposomal doxorubicin in the management of ovarian cancer. Ther Clin Risk Manag 6, 463-483, 2010.

Gao G, Ze Y, Li B, Zhao X, Zhang T, Sheng L, Hu R, Gui S, Sang X, Sun Q, Cheng J, Cheng Z, Wang L, et al. Ovarian dysfunction and gene-expressed characteristics of female mice caused by long-term exposure to titanium dioxide nanoparticles. J Hazard Mater 243, 19-27, 2012.

Geraets L, Oomen AG, Krystek P, Jacobsen NR, Wallin H, Laurentie M, Verharen HW, Brandon EF, de Jong WH. Tissue distribution and elimination after oral and intravenous administration of different titanium dioxide nanoparticles in rats. Part Fibre Toxicol 11, 30, 2014.

Grande F, Tucci P. Titanium dioxide nanoparticles: a risk for human health? Mini Rev Med Chem 16, 762-769, 2016.

Hong FS, Yu XH, Wu N, Zhang YQ. Progress of in vivo studies on the systemic toxicities induced by titanium dioxide nanoparticles. Toxicol Res 6, 115-133, 2017.

Hou CC, Zhu JQ. Nanoparticles and female reproductive system: how do nanoparticles affect oogenesis and embryonic development. Oncotarget 8, 109799-109817, 2017.

Iavicoli I, Leso V, Fontana L, Bergamaschi A. Toxicological effects of titanium dioxide nanoparticles: a review of in vitro mammalian studies. Eur Rev Med Pharmacol Sci 15, 481-508, 2011.

Iavicoli I, Leso V, Bergamaschi A. Toxicological effects of titanium dioxide nanoparticles: a review of in vivo studies. J Nanomater 2012, 964381, 2012.

Iavicoli I, Fontana L, Leso V, Bergamaschi A. The effects of nanomaterials as endocrine disruptors. Int J Mol Sci 14, 16732-16801, 2013.

Kolena J, Scsukova S, Jezova M, Furdova J, Tatara M, Jasem P. Effect of phospholipids on the reconstitution and thermal stability of delipidated rat ovarian luteinizing hormone/human chorionic gonadotropin receptors in proteoliposomes. Mol Cell Endocrinol 113, 53-60, 1995.

Larson JK, Carvan MJ 3rd, Hutz RJ. Engineered nanomaterials: an emerging class of novel endocrine disruptors. Biol Reprod 91, 20, 2014.

Moritz M, Gezske-Moritz M. Recent developments in the application of polymeric nanoparticles as drug carriers. Adv Clin Exp Med 24, 749-758, 2015.

Newbold RR, Jefferson WN, Padilla-Banks E. Long-term adverse effects of the neonatal exposure to bisphenol A on the murine female reproductive tract. Reprod Toxicol 24, 253-258, 2007.

Philbrook NA, Winn LM, Afrooz AR, Saleh NB, Walker VK. The effect of TiO(2) and Ag nanoparticles on reproduction and development of Drosophila melanogaster and CD-1 mice. Toxicol Appl Pharmacol 257, 429-436, 2011. 
Picut CA, Dixon D, Simons ML, Stump DG, Parker GA, Remick AK. Postnatal ovary development in the rat: morphologic study and correlation of morphology to neuroendocrine parameters. Toxicol Pathol 43, 343-353, 2015.

Rollerova E, Jurcovicova J, Mlynarcikova A, Sadlonova I, Bilanicova D, Wsolova L, Kiss A, Kovriznych J, Kronek J, Ciampor F, Vavra I, Scsukova S. Delayed adverse effects of neonatal exposure to polymeric nanoparticle poly(ethylene glycol)-block-polylactide methyl ether on hypothalamic-pituitary-ovarian axis development and function in Wistar rats. Reprod Toxicol 57, 165-175, 2015a.

Rollerova E, Tulinska J, Liskova A, Kuricova M, Kovriznych J, Mlynarcikova A, Kiss A, Scsukova S. Titanium dioxide nanoparticles: some aspects of toxicity/focus on the development. Endocr Regul 49, 97-112, $2015 \mathrm{~b}$.

Rompelberg C, Heringa MB, van Donkersgoed G, Drijvers J, Roos A, Westenbrink S, Peters R, van Bemmel G, Brand W, Oomen AG. Oral intake of added titanium dioxide and its nanofraction from food products, food supplements and toothpaste by the Dutch population. Nanotoxicology 10, 1404-1414, 2016.

Sebekova K, Dusinska M, Simon Klenovics K, Kollarova R, Boor P, Kebis A, Staruchova M, Vlkova B, Celec P, Hodosy J, Baciak L, Tuskova R, Beno M, Tulinska J, Pribojova J, Bilanicova D, Pojana G, Marcomini A, Volkovova K. Comprehensive assessment of nephrotoxicity of intravenously administered sodium-oleate-coated ultrasmall superparamagnetic iron oxide (USPIO) and titanium dioxide (TiO2) nanoparticles in rats. Nanotoxicology 8, 142-157, 2014.

Semete B, Booysen L, Lemmer Y, Kalombo L, Katata L, Verschoor J, Swai HS. In vivo evaluation of the biodistribution and safety of PLGA nanoparticles as drug delivery systems. Nanomedicine 6, 662-671, 2010.

Shen S, Du XJ, Liu J, Sun R, Zhu YH, Wang J. Delivery of bortezomib with nanoparticles for basal-like triple-negative breast cancer therapy. J Control Release 208, 14-24, 2015.

Shi HB, Magaye R, Castranova V, Zhao JS. Titanium dioxide nanoparticles: A review of current toxicological data. Part Fibre Toxicol 10, 15, 2013.

Shin HC, Alani AWG, Rao DA, Rockich NC, Kwon GS. Multi-drug loaded polymeric micelles for simultaneous delivery of poorly soluble anticancer drugs. J Control Release 140, 293-300, 2009.

Shin HC, Cho H, Lai TC, Kozak KR, Kolesar JM, Kwon GS. Pharmacokinetic study of 3-in-1 poly(ethylene glycol)block-poly(D,L-lactic acid) micelles carrying paclitaxel, 17-allylamino-17-demethoxygel danamycin, and rapamycin. J Control Release 163, 93-99, 2012.

Scsukova S, Mlynarcikova A, Kiss A, Rollerova E. Effect of polymeric nanoparticle poly(ethylene glycol)-blockpoly(lactic acid) (PEG-b-PLA) on in vitro luteinizing hormone release from anterior pituitary cells of infantile and adult female rats. Neuro Endocrinol Lett 36, 88-94, 2015.

Scsukova S, Bujnakova Mlynarcikvoa A, Kiss A, Rollerova E. Adverse eff ects of polymeric nanoparticle poly(ethylene glycol)- block-polylactide methyl ether (PEG-b-PLA) on steroid hormone secretion by porcine granulosa cells. Endocr Regul 51, 96-104, 2017.

Stefansdottir A, Fowler PA, Powles-Glover N, Anderson RA, Spears N. Use of ovary culture techniques in reproductive toxicology. Reprod Toxicol 49, 117-135, 2014.

Tassinari R, Cubadda F, Moracci G, Aureli F, D’Amato M, Valeri M, De Baerardis B, Raggi A, Mantovani A, Passeri D, Rossi M, Maranghi F. Oral, short-term exposure to titanium dioxide nanoparticles in Sprague-Dawley rat: focus on reproductive and endocrine systems and spleen. Nanotoxicology 8, 654-662, 2014.

Taylor U, Barchanski A, Kues W, Barcikowski S, Rath D. Impact of metal nanoparticles on germ cell viability and functionality. Reprod Domest Anim 47, 359-368, 2012.

Wang A, Marinakos SM, Badireddy AR, Powers ChM, Keith AH. Characterization of physicochemical properties of nanomaterials and their immediate environments in high-throughput screening of nanomaterial biological activity, WIREs Nanomed Nanobiotechnol 5, 430-448, 2013.

Wang N, Wang Z, Nie S, Song L, He T, Yang S, Yang X, Yi C, Wu Q, Gong C. Biodegradable polymeric micelles coencapsulating paclitaxel and honokiol: a strategy for breast cancer therapy in vitro and in vivo. Int J Nanomedicine 12, 1499-1514, 2017.

Weir A, Westerhoff P, Fabricius L, Hristovski K, von Goetz N. Titanium dioxide nanoparticles in food and personal care products. Environ Sci Technol 46, 2242-2250, 2012.

Xiao RZ, Zeng ZW, Zhou GL, Wang JJ, Li FZ, Wang AM. Recent advances in PEG-PLA block copolymer nanoparticles. Int J Nanomedicine 5, 1057-1065, 2010.

Xiao L, Xiong X, Sun X, Zhu Y, Yang H, Chen H, Gan L, Xu H, Yang X. Role of cellular uptake in the reversal of multidrug resistance by PEG-b-PLA polymeric micelles. Biomaterials 32, 5148-5157, 2011.

Zhang X, Li W, Yang Z. Toxicology of nanosized titanium dioxide: an update. Arch Toxicol 89, 2207-2217, 2015.

Zhao X, Ze Y, Gao G, Sang X, Li B, Gui S, Sheng L, Sun Q, Cheng J, Cheng Z, Hu R, Wang L, Hong F. Nanosized TiO2induced reproductive system dysfunction and its mechanism in female mice. PLoS ONE 8, e59378, 2013. 